\title{
Fatigue crack surface area and crack front length: new ways to look at fatigue crack growth
}

\author{
Jesse van Kuijk ${ }^{1, *}$, René Alderliesten ${ }^{1}$, Rinze Benedictus ${ }^{1}$ \\ ${ }^{1}$ Structural Integrity \& Composites, Faculty of Aerospace Engineering, TU Delft, Netherlands
}

\begin{abstract}
This paper discusses the appropriateness of crack length as a reference dimension for fatigue damage. Current discussion on short crack versus long crack data is still divided between various approaches to model small crack growth. A proper physical explanation of the probable cause of the apparent differences between short crack and long crack data is not yet provided. Long crack data often comprises crack growth in constant thickness specimens, with a through crack of near constant crack front geometry. This is not true for corner cracks or elliptical surface crack geometries in the small crack regime where the crack front geometry is not symmetric or through-thickness. This affects similitude parameters that are based on the crack length. The hypothesis in this paper is that a comparison between long crack data and short crack data should be made using similar increments in crack surface area. The work applied to the specimen is dissipated in generation of fracture surface, whereas fracture length is a result. The crack surface area approach includes the two-dimensional effect of crack growth geometry in the small crack regime. A corner crack and a through crack are shown to follow the same power law relationship when using the crack area as base parameter. The crack front length is not constant, and its power law behaviour for a corner crack is shown.
\end{abstract}

\section{List of Symbols}

$\Delta K_{a} \quad$ : Stress intensity factor, $\mathrm{f}(a)$

$\Delta K_{t h} \quad:$ Threshold stress intensity factor, $\mathrm{f}(a)$

$\Delta K_{A} \quad:$ Stress intensity factor, $\mathrm{f}(A)$

$\beta \quad$ : Elliptical aspect ratio modifier factor

$\gamma \quad:$ Aspect ratio of ellipse; $=b / a$

a : Crack length

$b \quad$ : Crack length through thickness

$d a / d N \quad:$ Crack length growth rate per cycle

$d A / d N \quad$ : Crack area growth rate per cycle

$f \quad$ : Crack front length

$A \quad$ : Crack area

$N \quad$ : Number of cycles or iterations

$R \quad$ : Stress ratio

$S_{\max } \quad:$ Maximum applied stress

$S_{o l} \quad$ : Maximum applied overload stress

$T \quad$ : Specimen thickness

$W \quad$ : Specimen width

\section{Introduction}

Most fatigue crack growth data is published as $d a / d N$ versus $\Delta K .^{\text {a }}$ The data often appears as one or more straight lines in a graph with log-log scales, and is known as the 'Paris law'. In industry, this Paris relation is widely used for its simplicity. However, small crack data is not so well-defined and a mismatch with Paris relations is often seen in this region.

Alderliesten [1,2] questions the general idea that the Paris relation in all its forms should be taken as a 'law', as there is no physical basis for the power law. He suggests that a better understanding of fatigue crack growth could be obtained by looking at the energy balance throughout fatigue cycles. Amsterdam et al. [3] agree with the notion that the power law approach is flawed from a physical point of view, as the equation is not dimensionally correct.

The relationship of $d a / d N$ versus $\Delta K_{a}$ on the 'base parameter' crack length $a$ seems to work fine for through cracks, where the crack front is ideally straight, and perpendicular to side of the specimen. For any other crack front shape, this does not necessarily hold. The mean $a$ along the crack front is different from the

\footnotetext{
${ }^{a}$ As the stress intensity factor is a function of the crack length, the notation $\Delta K_{a}$ will be used to distinguish it from another stress intensity factor introduced in this paper being a function of the crack area: $\Delta K_{A}$.
} 
observed $a$ at the specimen edge, and different local $d a / d N$ values are present along the crack front. Another choice of base parameter might improve the understanding of fatigue crack growth, and could help in understanding the energy balance cycle during crack growth.

\section{Hypothesis}

Given the large spread in small crack $d a / d N$ data, and the dependency on $a$ which is questionable for several crack front geometries, further search for a more suitable base parameter seems warranted.

It is hypothesized that the crack area $A$ is a better choice than the crack length $a$, because the area can be related to the energy in the cross-section, providing a path to relate the energy input to the crack growth. A schematic view of a specimen cross-section with cracked surface and geometric parameters is shown in Fig. 1.

During crack growth the cross-sectional area decreases, whereas the crack length is a result of this area decrease along one dimension. Only for an ideal through crack with straight crack front are $a$ and $A$ directly related by the specimen thickness. With literature mostly dealing with through cracks and $a$ being readily measurable from the specimen surface, it is understandable why $a$ is such a popular parameter to describe crack growth (rate).

Note that potential drop measurements are essentially crack area measurements, as the area is related to the electrical resistance of the current. When the area measurement is normalised by the specimen thickness $T$ it directly transforms into a mean $a$ measurement.

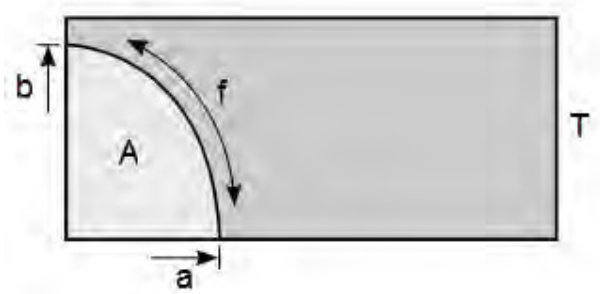

Fig. 1. Schematic view of a specimen cross-section with cracked surface $A$ and thickness $T$. Crack lengths $a$ and $b$ are indicated, as well as crack front length $f$.

With $A$ as base parameter, it is straightforward that $d A / d N$ becomes the crack growth parameter. Related to this choice is the suitability of similitude parameter $\Delta K_{a}$. To comply with the choice of $A$ as base parameter, an equivalent similitude parameter $\Delta K_{A}=\Delta S \sqrt{\pi A}$ is used. Again, this change would hardly affect through-cracks, or the through-crack phase of other crack types.

A corner crack grows along two dimensions ( $a$ and $b$ ) instead of one $(a)$, and as such the crack front length $f$, measured from free surface to free surface along a crack front, is also growing per cycle. It is hypothesized that this lengthening of $f$ can be correlated to $d a / d N$ or $d A / d N$ as well, as the available energy for crack growth per cycle is divided over $d a$ and $f$.

\section{Examples from literature}

Two examples from literature are shown. The first contains data from fatigue tests, while the second presents a common analytical corner crack model.

\subsection{Corner cracks in PMMA}

There is ample literature on fatigue crack growth rate $d a / d N$ versus $\Delta K_{a}$ data. Very few include crack front geometry data. Grandt et al. [4] discuss various corner crack and surface crack tests by Snow [5] where the crack front geometry was recorded during the test. An example is given in Fig. 2. From this data both $a$ and $A$ can be obtained. The number of analysed crack fronts and data points per front are rather limited, but it provides insight into the change of $a$ and $A$ during the growth of a corner crack.

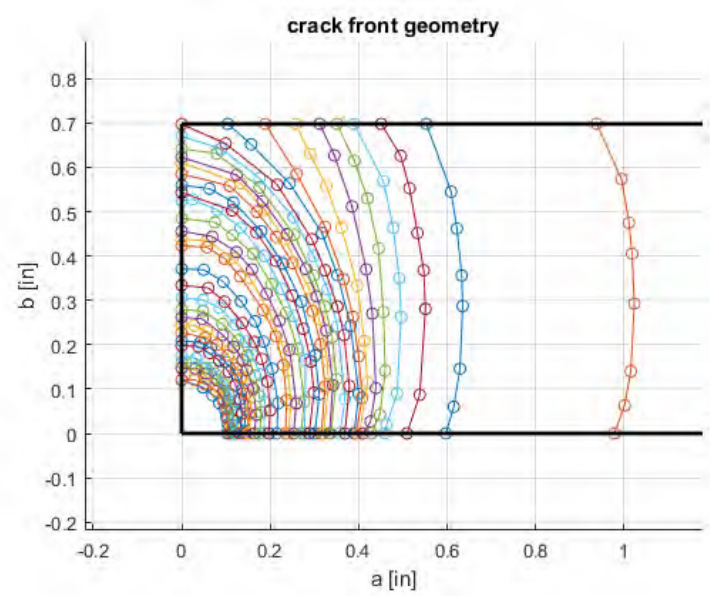

Fig. 2. Corner crack geometry of several crack fronts of Test 6 by Snow [5]. Measurement points belonging to identical crack fronts have been joined by lines for clarity.

The corresponding $d a / d N$ versus $\Delta K_{a}$ plot is given in Fig. 3. The scatter in the corner phase is evident, and when the crack becomes a through crack, the curve becomes a smooth power law. From textbook crack growth curves, one would expect a through crack to have a steeper curve during small crack growth, starting at a $\Delta K_{t h}$ (e.g. Schijve [6], Fig. 8.6). For the corner crack shown in Fig. 3, the opposite is seen. 


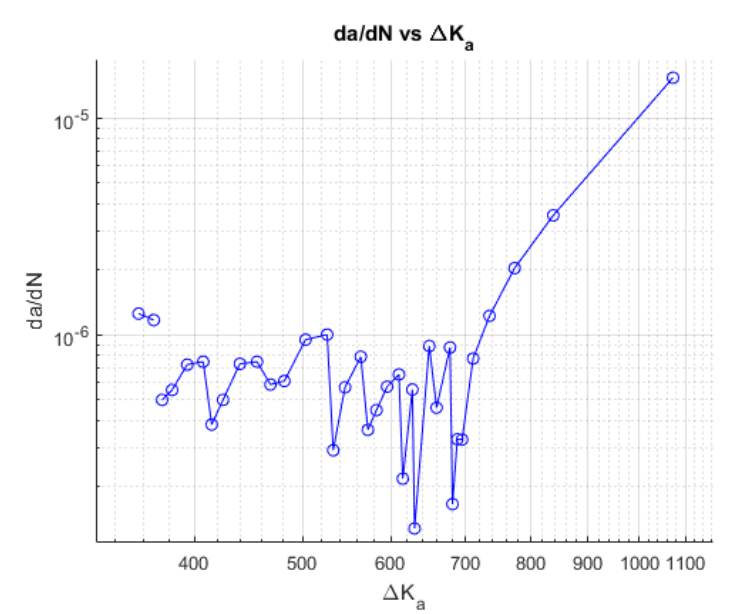

Fig. 3. $d a / d N$ versus $\Delta K_{a}$ belonging to Test 6 by Snow [5].

\subsection{Corner crack model of Newman and Raju}

A simple model of a corner crack is presented by Newman and Raju [7]. A quarter ellipse corner crack is modelled, after which it becomes instantly a through crack. There is no transition phase between the cornerand through crack phases, and the model is based on the crack length throughout all phases; which gives the false impression that the $d a / d N$ curve is smooth. Fig. 4 shows this transition at $a / T=0.675$. This model is of interest, however, because a good comparison example is given with experimental data of Hsu et al. [8].

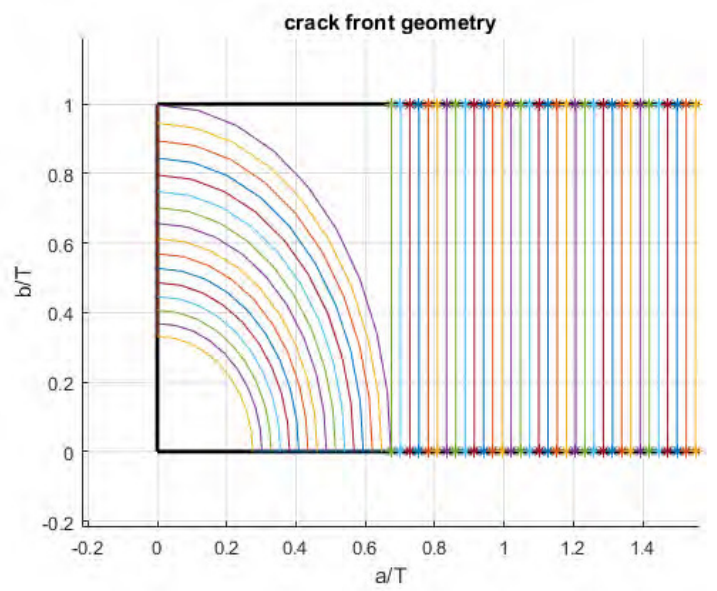

Fig. 4. Model of a corner crack. Redrawn from Newman and Raju [7]. With $a$ as base parameter, $d a / d N$ appears smooth while there is a large, unrealistic step in $A$ at $a / T=0.675$.

\section{Numerical modelling}

Two numerical models are presented here that are used to predict crack front geometries during crack growth.

\subsection{Corner crack and through crack comparison model}

The premise here is that $d A / d N$ versus $\Delta K_{A}$ is a power law. $A$ could be a more suitable parameter for comparison of several crack types, and it would still compare with $d a / d N$ versus $\Delta K_{a}$ for the through crack.
A model was created which simulates a corner crack, together with an equivalent through crack. Although the appearance of the corner crack might be similar to the previously discussed Newman-Raju model, it is partially more detailed as the three phases are modelled separately. By modelling the transition region with a smooth $d A / d N$, the unrealistic behaviour of the Newman-Raju model (a significant $d A$ between two cycles, both at identical $a$ ) is largely mitigated. Furthermore the crack length $a$ or crack growth ratio $d a / d N$ is not used as base parameter, but $d A / d N$ is.

The corner crack is modelled as a quarter ellipse crack front, whose aspect ratio (minor axis over major axis) $\gamma$ is varied exponentially by a factor $\beta$ during growth to mimic crack behaviour seen in literature and in reality. Per iteration $N, \gamma$ is multiplied by $\beta^{N}$. The area of a quarter ellipse area is $A=\pi a b / 4$. With $b=a \gamma$, reworking gives $a=\sqrt{4 A /(\pi \gamma)}$, and $a$ and $b$ are obtained. Then $b$ is calculated using $a$ and $\gamma$. Input consists of specimen width, thickness, and the stress range $^{\mathrm{b}} \Delta S$. The values of $d A / d N$ and $\beta$ are given, and the model grows a corner crack until it becomes throughthickness; at $b \geq T$.

For the transition phase, a given number of cycles is chosen such to represent real crack front development when transforming a corner crack into a through crack. The $d A / d N$ power law to drive the area growth is still used, while graphically the crack is deformed from a quarter ellipse to a straight line, by flattening the elliptical curve gradually into a straight line, i.e. it takes account of the growth of $d A$ and $A$ but does only approximate the crack front development graphically.

The model then also simulates a true through crack for a given number of cycles, matching up with the through crack phase from the corner crack model.

For two cases, each containing a corner and a through crack, the $d a / d N$ versus $\Delta K_{a}$ and $d A / d N$ versus $\Delta K_{A}$ are obtained, and discussed below. An example of the crack geometry of the first case is given in Fig. $\mathbf{5}$.

\footnotetext{
${ }^{\mathrm{b}}$ Strictly speaking, $\Delta S$ is a constant here, and can be set to unity. As the model does not take into account the applied stress or load cycles, an $R$-effect is not present.
} 


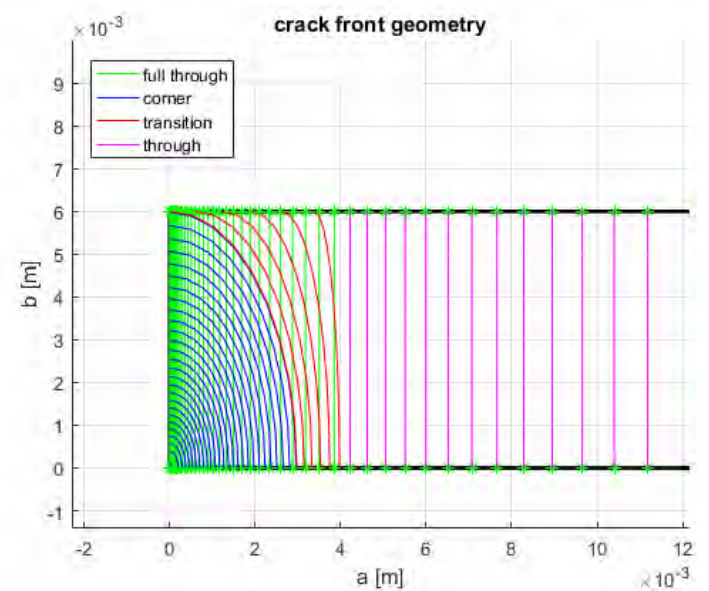

Fig. 5. A corner crack in three phases, with an overlaid true through crack, both following the same $d A / d N$ power law.

The $d a / d N$ data from this model is presented in Fig. 6. It is not unreasonable as it correlates to real results, e.g., Fig. 2. When looking at the $d A / d N$ data in Fig. 7, the values are found to be continuous and increasing all the time. There is some slight deviation from the linear power law behaviour in this log-log plot, in the very beginning of the crack growth. This is because the power law $^{c} \mathrm{f}(x)=a x^{b}+c$, has $c \neq 0$ because of a finite starting crack area present. Nevertheless it is evident that various crack geometries can be compared much better when based on $A$ instead of $a$.

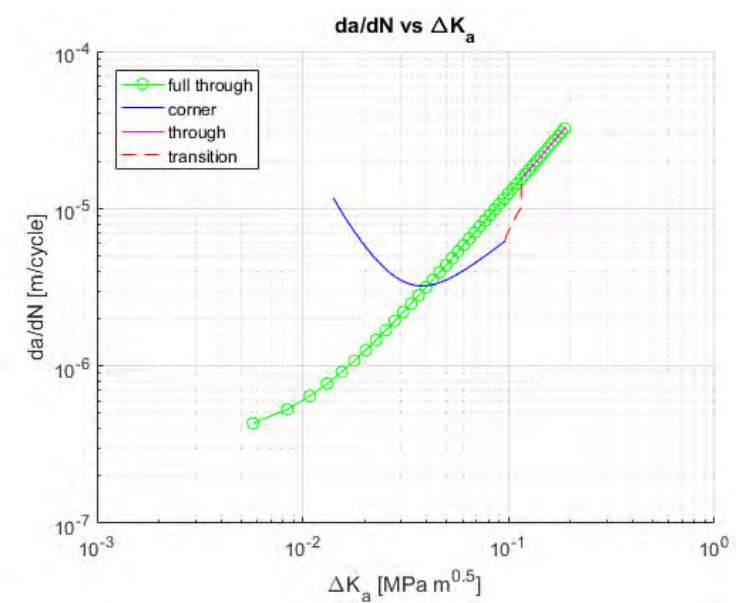

Fig. 6. The standard $d a / d N$ versus $\Delta K_{a}$ curve of this model. Note how irregular the corner and transition phases behave, while the true through crack is faithful to the power law.

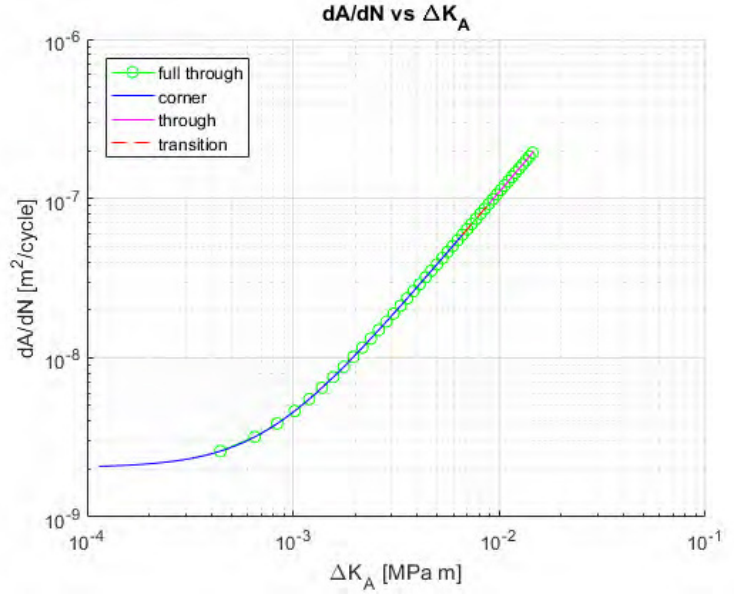

Fig. 7. When presenting the crack growth data as $d A / d N$ versus $\Delta K_{A}$, both crack types predictably collapse onto the same power law (since it was programmed to follow this relation), even though they are geometrically different.

Slight changes in starting parameters (mainly constant $\beta$, which acts as an exponential function on $\gamma$ ), can alter the ellipse aspect ratio $\gamma$ during crack growth such that the $d a / d N$ actually decreases during the corner crack phase. It increases again through the transition and through crack phases. This is the second case. An example of that is given in Fig. 9, together with the crack front geometry in Fig. 8. The $d A / d N$ data of this case is equal to the data shown in Fig. 7.

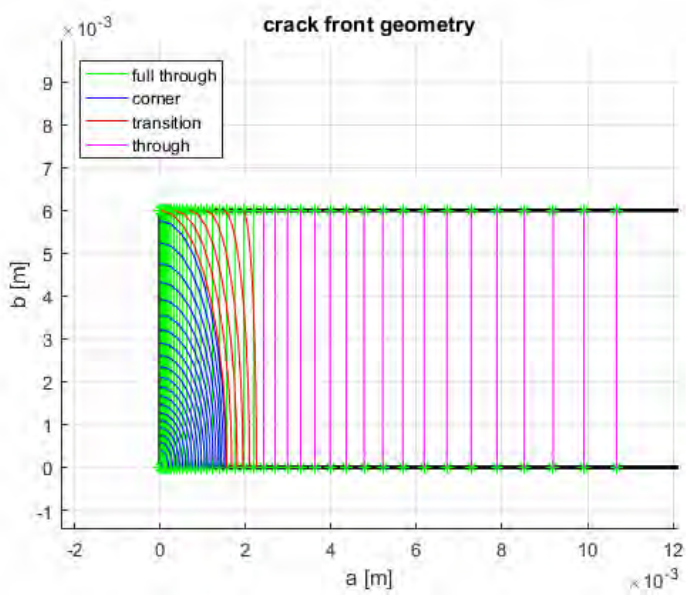

Fig. 8. A corner crack in three phases, with an overlaid true through crack, both following the same $d A / d N$ power law.

\footnotetext{
${ }^{\mathrm{c}}$ In this example equation, $a, b$, and $c$, are generic constants, with $x$ a generic variable.
} 


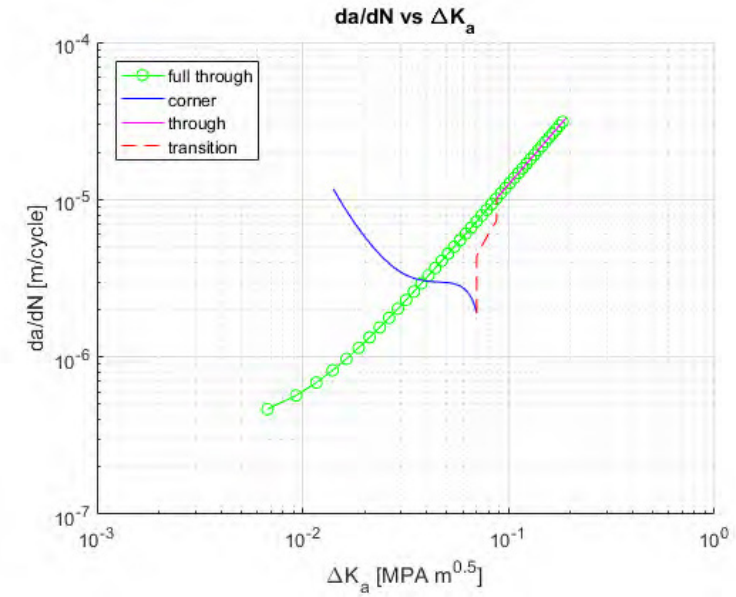

Fig. 9. The standard $d a / d N$ versus $\Delta K_{a}$ curve of this model. Note how irregular the corner and transition phases behave, while the true through crack is faithful to the power law.

\subsection{Cellular Automaton}

A cellular automaton procedure was programmed to predict crack front growth, based on unpublished work by Conen [9]. The specimen cross-section is modelled as a matrix. Every iteration $N$, all indices of a probability matrix are updated based on certain surrounding indices, and a binary version of this matrix is stored, indicating which part of the cross-sectional area is still solid and which part has disappeared (cracked). The crack fronts are generated every $N$, and as such do not follow any growth law. A crack growth relation is introduced separately to read out the correct crack fronts. A power law relation on $d A$ is chosen to obtain the growth intervals. From the data also the crack length $a$ is determined, to calculate $\Delta K_{a}$. Fig. 10 shows an example of a grown corner crack. (Note the similarity with Fig. 9). Fig. 11 gives the corresponding $d a / d N$ versus $\Delta K_{a}$.

Given the nature of the simulation, $d a / d N$ here appears also realistic, but given the model resolution, less smooth.

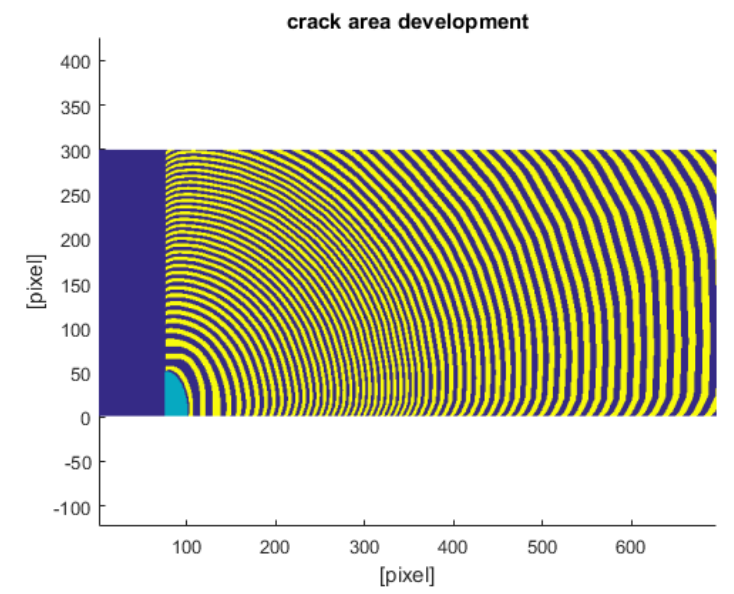

Fig. 10. Cellular Automaton has grown a corner crack, from left to right. Half of the centre hole is visible as the dark rectangle on the left, and the initial crack in turquoise. Dimensions in pixels.

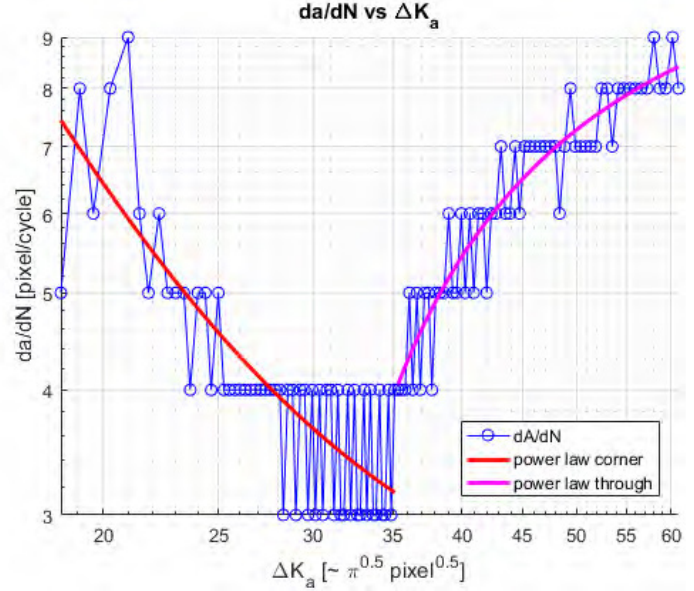

Fig. 11. The standard $d a / d N$ versus $\Delta K_{a}$ curve of this model. The corner phase clearly has a decreasing growth rate, an only after becoming through-thickness it assumes a normal growth behaviour.

And again, when plotting the growth with $A$ as base parameter, a smooth power law shows up: Fig. 12.

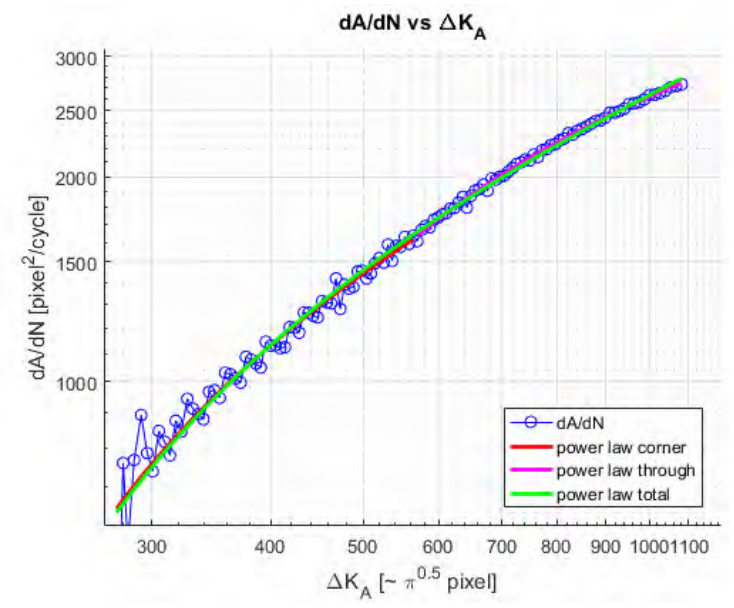

Fig. 12. When showing the crack growth data as $d A / d N$ versus $\Delta K_{A}$, both crack phases connect with nearly equal slopes The complete crack follows a power law relationship neatly.

To show the power of the cellular automaton, consider the example crack growth in Fig. 13. A corner crack is present, as well as a single slit radiating perpendicular from the centre hole. The zebra-striped pattern shows clearly how the cracks start to grow independently, and then link up to form one crack front. 


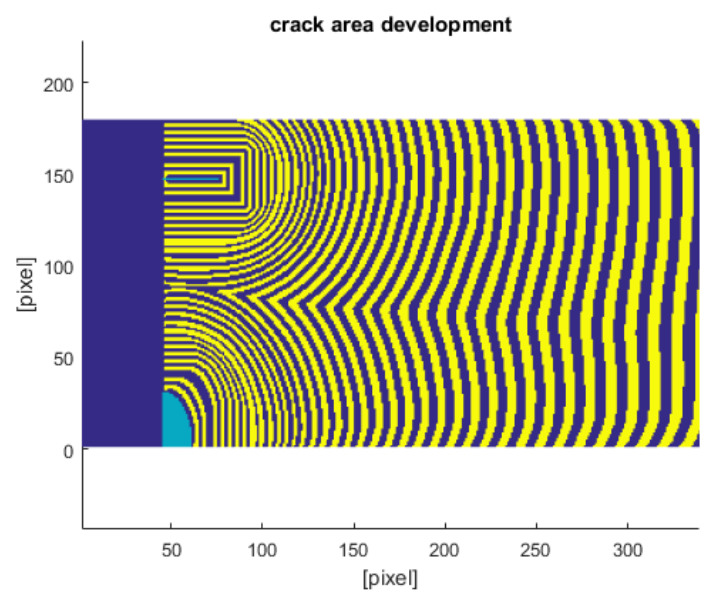

Fig. 13. A corner crack and a slit crack (both in turquoise) are grown. Note how the crack fronts join and become one through crack.

\section{Crack front length}

Another parameter often overlooked in (small) crack literature is the crack front length $f$. There is a marked increase and decrease of $f$ in respectively the corner and transition phase. Energy is used to increase the crack surface area along two dimensions here, which also modifies $f$. For an ideal through-crack, only the surface area is increased, and $f$ is constant.

When plotting the crack front length development for the literature case of Grandt et al. [4] in Fig. 14 again a power law relationship is found for the corner phase. The decrease in the transition phase is small, and not enough data points are available to make reliable curve fit estimations. The power law is not a surprise, as it follows from the power law dependency of $d A / d N$.

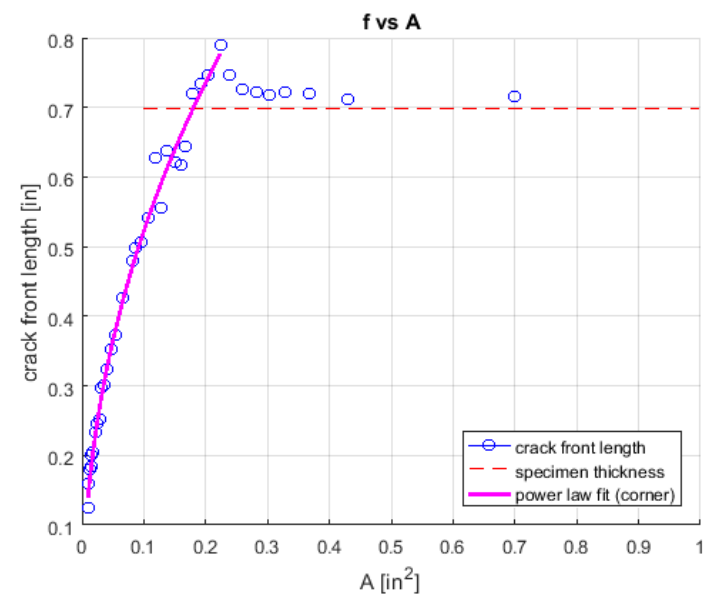

Fig. 14. Snow Test 6 [5]. $f$ increases until the crack becomes a through crack, after which it decreases to a constant value. This end value is slightly larger than the specimen thickness since the crack front is slightly curved. Some data scatter is present.

Evaluating the crack front lengths of the cases presented in Section 4.1, very good power law fits are found for both the corner and transition phases, as shown in Fig. 15 and Fig. 16.

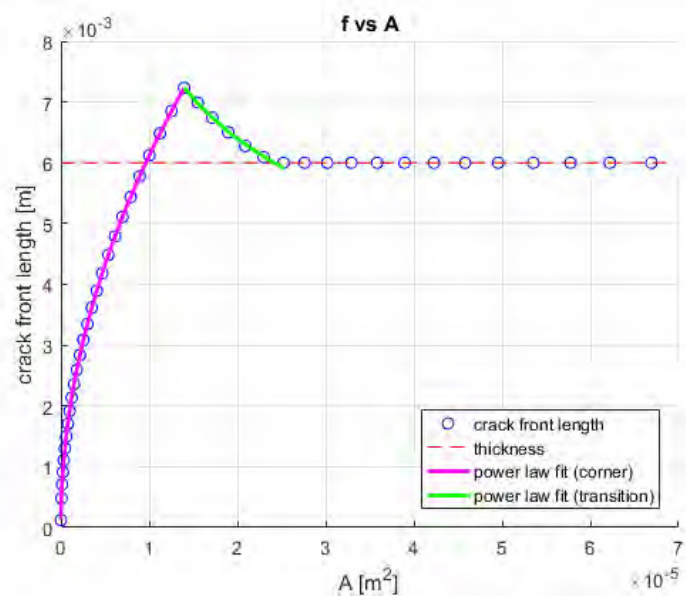

Fig. 15. Crack front length plot belonging to Fig. 5. $f$ increases with a near perfect power law fit until becoming a through crack. Beyond that, $f$ decreases to the specimen thickness (straight through crack), again following a power law.

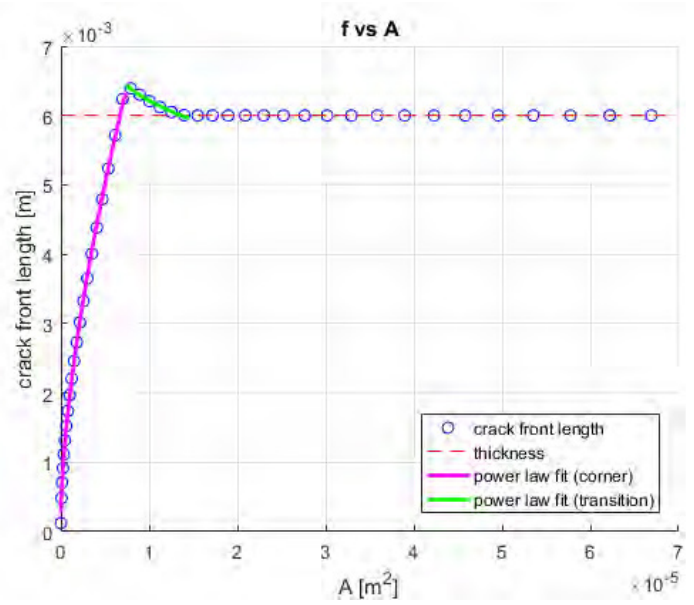

Fig. 16. Crack front length plot belonging to Fig. 8. $f$ increases with a near perfect power law fit until becoming a through crack. Beyond that, $f$ decreases to the specimen thickness (straight through crack), again following a power law.

This power law relation for $f$ versus $A$ also holds in the cellular automaton simulation, see Fig. 17. Only here a small step in absolute value is seen when the crack goes 'around the corner'. The crack is not exactly a quarter-ellipse, so some crack length is lost in the very corner at $b=T$. This is visible from the yellow markings in the top left corner in Fig. 10. Beyond the corner phase, the transition phase asymptotically nears a real through crack phase, but again with a good power law fit. 


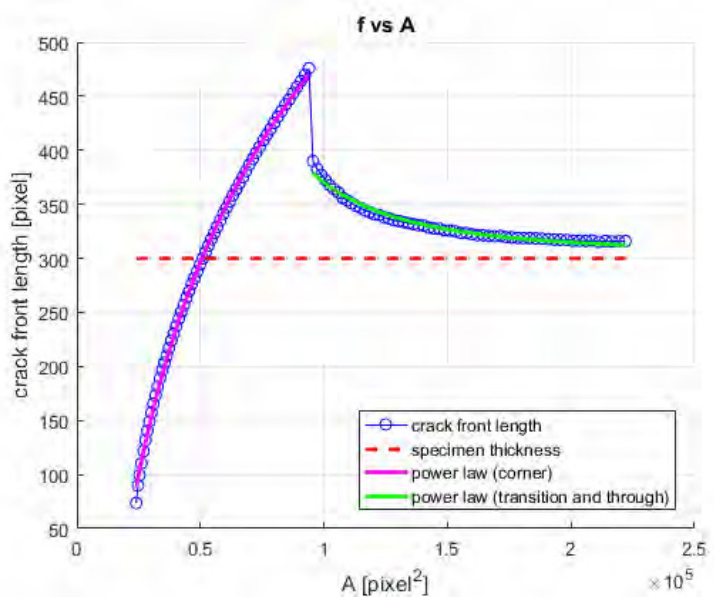

Fig. 17. Cellular automaton: $f$ increases with a near perfect power law fit until becoming a through crack. Beyond that, $f$ decreases asymptotically to a value slightly larger than the specimen thickness (curved through crack), again following a power law. See also Fig. 10.

\section{Discussion}

The figures from Grandt et al. [4] are very illustrative. However, these cracks were grown in PMMA material, a polymer. Most fatigue crack growth data in literature is gathered from metals (aluminium/steel/titanium), which tend to be more isotropic than a polymer. Nevertheless these results are very similar to the results found in metals. The crack growth mechanism for cracks at this scale might not be very sensitive to anisotropic material structures.

Given the behaviour of $d a / d N$ versus $\Delta K_{a}$ as seen in Fig. 6 and Fig. 9, the use of a Paris relationship for a corner crack is incorrect. The model of the author (Section 4.1) does not show such a change in slope when using $d A / d N$ versus $\Delta K_{A}$, see Fig. 7. However, introducing a different crack growth rate power law for the small crack regime in this model does not make it exactly match up with corner crack growth rate curves either, although close. The geometry effect can significantly affect the crack growth rate in the small crack growth regime.

Furthermore, consider a surface crack inside a hole in a plate. Such a crack also grows through the three phases. In the first phase, by definition, $a$ is constant as the crack first has to grow to a through-thickness crack. In the standard $d a / d N$ versus $\Delta K_{a}$ plot, $d a / d N=0$ here, such that these points cannot be shown on the log$\log$ plot. The $\Delta K_{a}$ parameter is constant during this phase, while the crack is growing. This shows up as a $\Delta K_{t h}$, while the second dimension is not present in this similitude parameter.

The cellular automaton is an interesting method, in the sense that it has absolutely no physical connection with fatigue crack growth, yet it produces eerily similar crack growth geometries. Two major arguments can be made against using this method:

- The growth $d A / d N$ is artificially and a-priori introduced using a power law. The automaton grows crack fronts, but lacks a coupling with the number of cycles. The relevant crack fronts are found using an area-related relationship.

- The growth rate along a single crack front is not constant, but the variation in local stress intensity factors, linked to local growth rate, seems to be smaller than observed in reality.

Regarding the latter argument, the Newman-Raju model and the author's model correct slightly for this by changing $\gamma$ of the ellipse during growth, but do limit the shape to the ellipse. The cellular automaton tends to make crack fronts slightly too curved or too much alike a circular arc compared to reality, but captures the transition region wonderfully realistic.

While these arguments are valid concerns, the results do appear similar to geometries observed in practice and reported in literature.

\section{Conclusions and recommendations}

The choice of crack length $a$ as base parameter for fatigue crack growth measurements is questionable for crack types other than through cracks. It is shown that corner cracks behave differently in the small crack growth region. The crack surface area $A$ is shown to be a more suitable parameter, as the results for both through cracks and corner cracks are now similar in magnitude and slope on a graph with log-log scales, making comparisons easier. This change in base parameter affects the similitude parameter too. The common $d a / d N$ versus $\Delta K_{a}$ plot can then be transformed into an equivalent $d A / d N$ versus $\Delta K_{A}$.

It is shown that in a quarter-elliptical corner crack, the crack front length during the corner phase grows along a similar power law behaviour as introduced for $d A$. The energy used for crack growth is basically split into crack area increase and crack front length extension.

It is shown that a corner crack has three distinct phases; corner, transition, and through crack. The crack length growth rate behaves markedly different from a power law during the corner phase, and the transition phase links the corner and through phases together.

The cellular automaton approach can create great insight in the behaviour of the crack growth, especially when unique and/or multiple initial cracks are present. Although the automaton routine has no physical link to fatigue crack growth, it can be used as a prediction tool for fatigue crack growth geometry, especially when multiple starter cracks are present.

As there is scarcely crack growth data available which also includes the crack front geometry development, more fatigue tests are needed with high accuracy measurements of the crack front geometry. This has proven to be difficult but not impossible, and warrants further study to create data sets to compare the models with.

Another recommendation is to include more fatigue crack types into the models, to see if the results for the corner crack versus through crack also hold for these types. 
This research was carried out under project number S21.5.15581 in the framework of the Partnership Program of the Materials innovation institute M2i (www.m2i.nl) and the Technology Foundation STW (www.stw.nl), which is part of the Netherlands Organisation for Scientific Research (www.nwo.nl).

\section{References}

1. R.C. Alderliesten, How proper similitude can improve our understanding of crack closure and plasticity in fatigue, Int. J. of Fatigue, 82 263-273 (2016)

2. R.C. Alderliesten, How proper similitude could have improved our understanding about fatigue damage growth, Proc. 28th ICAF Sym, 47-57 (2015)

3. E. Amsterdam, F. Grooteman, The influence of stress state on the exponent in the power law equation of fatigue crack growth. Int. J. of Fatigue, 82 572-578 (2016)

4. A.F. Grandt, J.A. Harter, D.E. Tritsch, Semielliptical cracks along holes in plates and lugs, AFWAL-TR83-3043 (1983)

5. J.R. Snow, A stress intensity factor calibration for corner flaws at an open hole, AFML-TR-74-282 (1975)

6. J. Schijve, Fatigue of Structures and Materials (2009)

7. J.C. Newman, I.S. Raju, Prediction of fatigue crackgrowth patterns and lives in three-dimensional cracked bodies, Proc. 6th Int. Conf. on Fracture (ICF6), 1597-1608 (1984)

8. T.M. Hsu, W.M. McGee, J.A. Aberson, Extended study of flaw growth at fastener holes, AFFDL-TR70-149 (1978)

9. M. Conen, Appendix B: Cellular Automator, unpublished work (2008) 